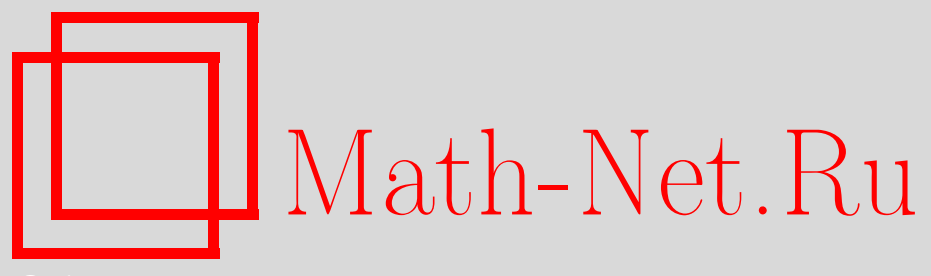

Ж. Бургейн, Я. Г. Синай, Предельное поведение больших чисел Фробениуса, УМH, 2007, том 62, выпуск 4, 7790

DOI: https://doi.org/10.4213/rm7150

Использование Общероссийского математического портала Math-Net.Ru подразумевает, что вы прочитали и согласны с пользовательским соглашением http: //www . mathnet.ru/rus/agreement

Параметры загрузки:

IP: 54.237 .206 .68

26 апреля 2023 г., 15:19:49

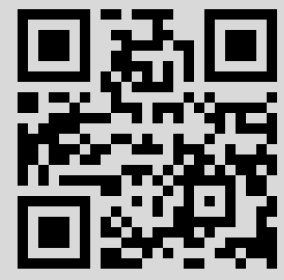




\section{Предельное поведение больших чисел Фробениуса}

\section{Ж. Бургейн, Я. Г. Синай}

В работе рассматривается задача об асимптотическом распределении чисел Фробениуса для $n$ взаимно простых чисел. Для $n=3$ получены практически окончательные результаты. Для $n>3$ показано, что возникающие распределения образуют компактное множество. Существенную роль играет предельная теорема для логарифмов знаменателей цепных дробей случайных чисел.

Библиография: 6 названий.

\section{СОДЕРЖАНИЕ}

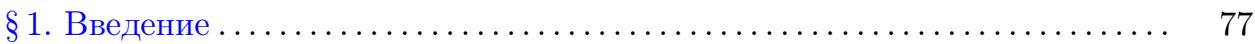

$\S 2$. Случай $n=3$ и нод $\left(a_{i}, a_{j}\right)=1$ для некоторых $a_{i}, a_{j} \ldots \ldots \ldots \ldots \ldots \ldots . \ldots . \ldots$

$\S 3$. Случай $n=3$ и произвольных $b_{i j}=$ нод $\left(a_{i}, a_{j}\right) \ldots \ldots \ldots \ldots \ldots \ldots \ldots . \ldots \ldots$

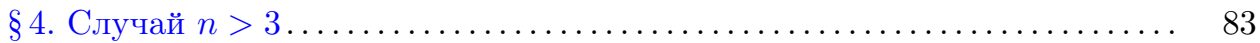

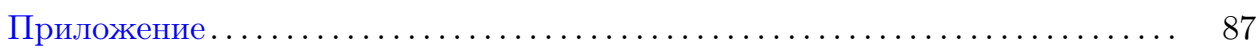

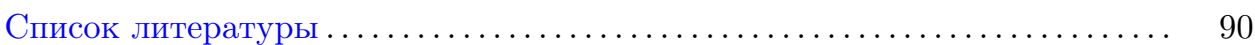

\section{$\S 1$. Введение}

Рассмотрим наборы $a=\left(a_{1}, a_{2}, \ldots, a_{n}\right)$ из $n$ положительных целых чисел, являющихся взаимно простыми, т. е. таких, что наибольший общий делитель (нод) всех $a_{j}$ равен 1 . Числом Фробениуса $F(a)$ набора $a$ называется наименьшее $F$ такое, что любое целое $t \geqslant F$ может быть записано в виде

$$
t=\sum_{j=1}^{n} x_{j} a_{j}
$$

с неотрицательными целыми $x_{j}$. В. И. Арнольд в [1] ввел ансамбль наборов $a$, для которых $a_{1}+a_{2}+\cdots+a_{n}=\sigma$ стремится к бесконечности, и изучил поведение $F(a)$ при $\sigma \rightarrow \infty$. В частности, он сформулировал гипотезу, согласно

Второй автор благодарит за финансовую поддержку фонд NSF (грант № 0600996).

(C) Ж. БУРГЕйн, Я.Г. СинАй, 2007 
которой для типичных $a F(a)$ растет как $\sigma^{1+\frac{1}{n-1}}$. Другие гипотезы и результаты Арнольда могут быть найдены в [2].

В этой работе мы рассматриваем другие ансамбли больших $a$ и изучаем тот же вопрос о росте $F(a)$ в этих ансамблях. А именно, фиксируем $N$ и обозначим через $\Omega_{N}$ множество всех $a$, для которых $1 \leqslant a_{j} \leqslant N, j=1,2, \ldots, n$, и нод $\left(a_{1}, a_{2}, \ldots, a_{n}\right)=1$. Используя элементарные вероятностные методы, легко показать, что $\lim _{N \rightarrow \infty} \frac{1}{N^{n}}\left|\Omega_{N}\right|$ существует и положителен (см., например, [3]). Тем самым задается "вероятность" набора $a \in \Omega_{N}$ в ансамбле всевозможных наборов $a$ из $n$ чисел, не превосходящих $N$. Ниже через $P_{N}$ обозначается равномерное распределение вероятностей на $\Omega_{N}$. Мы изучаем в этой работе поведение $F(a)$ для типичных $a$ (в смысле $P_{N}$ ) при $N \rightarrow \infty$. Случай $n=2$ легко следует из знаменитого результата Сильвестра о том, что $F(a)=\left(a_{1}-1\right)\left(a_{2}-1\right)$ (см. [4]). Из него вытекает, что $\frac{1}{N^{2}} F(a)$ имеет предельное распределение при $N \rightarrow \infty$.

Ниже в $\S 2$ и $\S 3$ мы рассматриваем случай $n=3$. Основываясь на некоторых фактах из теории цепных дробей, мы доказываем следующее утверждение.

ТеОРема 1. При $N \rightarrow \infty$ существует предельное распределение величинь $\frac{1}{N^{3 / 2}} F(a)$.

Доказательство необходимых фактов содержится в статье К. Ульчиграй и одного из нас (Я. Синая) [5]. Безнадежно пытаться выписать явно предельное распределение в теореме 1. Вероятно, методы этой работы могут быть использованы для оценки его убывания на бесконечности.

Ниже мы определяем другую функция $F_{1}(a)$, для которой доказываем лемму 1, показывающую, что в типичных ситуациях эта функция ведет себя так же, как $F(a)$. Но $F_{1}(a)$ намного легче для анализа, так как для нее основная проблема формулируется как "max-min"-проблема.

$\mathrm{B} \S 2$ мы обсуждаем случай $n=3$ при дополнительном условии, что нод $\left(a_{i}, a_{j}\right)$ равен единице по крайней мере для одной пары $a_{i}, a_{j}$. В $\S 3$ мы рассматриваем общий случай.

В $\S 4$ мы рассматриваем случай $n>3$. Теорема 2 этого параграфа показывает, что в модифицированных ансамблях величины $\frac{F(a)}{N^{1+\frac{1}{n-1}}}$ ограничены (равномерно по $N$ ) в том смысле, что

$$
P_{N, \alpha}\left\{\frac{F(a)}{N^{1+\frac{1}{n-1}}} \geqslant \mathcal{D}\right\} \leqslant \varepsilon(\mathcal{D}),
$$

где $\Omega_{N, \alpha}$ - ансамбль таких $a$, для которых $a_{j} \geqslant \alpha N, 1 \leqslant j \leqslant n$, и $P_{N, \alpha}-$ равномерное распределение в этом ансамбле, $0<\alpha<1$ - фиксированное число, $\varepsilon(\mathcal{D})$ не зависит от $N, \varepsilon(\mathcal{D}) \rightarrow 0$ при $\mathcal{D} \rightarrow \infty$. В приложении мы доказываем лемму, которая использовалась в более ранней версии этой работы и может иметь различные применения. Именно, мы показываем, что

$$
\ell\left\{\alpha: \sum_{m=1}^{M} \frac{1}{\left|e^{2 \pi i m \alpha}-1\right|} \geqslant \mathcal{D} M \ln M\right\} \leqslant \varepsilon_{1}(\mathcal{D}),
$$

где $\ell$ - мера Лебега на $[0,1], \varepsilon_{1}(\mathcal{D})$ не зависит от $M, \varepsilon_{1}(\mathcal{D}) \rightarrow 0$ при $\mathcal{D} \rightarrow \infty$. 
Анализ поведения выражения $\frac{1}{M} \sum_{m=1}^{M} \frac{1}{e^{2 \pi i m \alpha}-1}$ как функции от $M$ для типичных $\alpha$ важен в некоторых вопросах, но мы не будем этого касаться.

Теперь мы определим функцию $F_{1}(a)$ и докажем лемму 1 , показывающую, в каком смысле $F(a)$ и $F_{1}(a)$ эквивалентны. Для любого $a \in \Omega_{N}$ введем арифметическую прогрессию $\Pi_{r}=\left\{r+m a_{n}, m \geqslant 0\right\}, 0 \leqslant r<a_{n}$. Рассмотрим равенство

$$
x_{1} a_{1}+x_{2} a_{2}+\cdots+x_{n-1} a_{n-1}=r+m\left(x_{1}, \ldots, x_{n-1}\right) a_{n},
$$

из которого следует, что

$$
x_{1} a_{1}+x_{2} a_{2}+\cdots+x_{n-1} a_{n-1} \equiv r\left(\bmod a_{n}\right) .
$$

Здесь $x_{j} \geqslant 0$ - целые числа. Положим $\bar{m}_{r}=\min _{0 \leqslant x_{1}, \ldots, x_{n-1}<a_{n}} m\left(x_{1}, \ldots, x_{n-1}\right)$ и обозначим $F_{1}(a)=\max _{r}\left(r+\bar{m}_{r} a_{n}\right)$.

Лемма 1. $F_{1}(a)-a_{n} \leqslant F(a) \leqslant F_{1}(a)$ в предположении, что $F_{1}(a)-a_{n}>0$.

ДокАЗАТЕЛЬСтво. Возьмем $t \geqslant F_{1}(a)$. Тогда $t \in \Pi_{r}$ для некоторого $r$, $0 \leqslant r<a_{n}$, т. е. $t=r+m a_{n}$. Следовательно,

$$
t=r+\bar{m}_{r} a_{n}+\left(m-\bar{m}_{r}\right) a_{n}=x_{1} a_{1}+\cdots+x_{n-1} a_{n-1}+\left(m-\bar{m}_{r}\right) a_{n}
$$

для некоторых $0 \leqslant x_{j}<a_{n}, j=1, \ldots, n-1$. Так как $t \geqslant F_{1}(a)$, мы имеем $x_{1} a_{1}+\cdots+x_{n-1} a_{n-1} \leqslant F_{1}(a)$ и $m \geqslant \bar{m}_{r}$. Это дает необходимое представление $t$ и неравенство $F(a) \leqslant F_{1}(a)$.

Чтобы доказать неравенство с другой стороны, возьмем $r_{1}$ такое, что $F_{1}(a)=$ $r_{1}+\bar{m}_{r_{1}} a_{n}=\max _{r}\left(r+\bar{m}_{r} a_{n}\right)$. Мы покажем, что $t_{1}=r_{1}+\left(\bar{m}_{r_{1}}-1\right) a_{n}$ не может быть представлено в виде $t_{1}=y_{1} a_{1}+\cdots+y_{n} a_{n}$ с неотрицательными $y_{j}, 1 \leqslant j \leqslant n$. В самом деле, если бы такое представление было возможно, мы имели бы

$$
y_{1} a_{1}+\cdots+y_{n-1} a_{n-1}=r_{1}+m a_{n}
$$

для некоторого $m \geqslant \bar{m}_{r_{1}}$, а по определению

$$
t_{1}=y_{1} a_{1}+\cdots+y_{n-1} a_{n-1}+y_{n} a_{n}=r_{1}+m a_{n}+y_{n} a_{n}=r_{1}+\left(\bar{m}_{r_{1}}-1\right) a_{n} .
$$

Поэтому должно быть выполнено равенство $m+y_{n}=\bar{m}_{r_{1}}-1$. Но так как $m \geqslant \bar{m}_{r}$, это возможно, только если $y_{n}<0$. Лемма 1 доказана.

Конечно, вместо $a_{n}$ можно было бы взять любое другое $a_{j}$. В типичном случае мы ожидаем, что $x_{j}$ растут как $N^{\frac{1}{n-1}}$. Следовательно, в типичной ситуации $F(a) \sim F_{1}(a)$.

\section{§ 2. Случай $n=3$ и нод $\left(a_{i}, a_{j}\right)=1$ для некоторых $a_{i}, a_{j}$}

Без потери общности мы можем предположить, что $i=1, j=3$. В этом случае “max-min"-задача для $F_{1}(a)$ может быть решена более или менее явно. Напишем для положительных целых $x_{1}, x_{2}$

$$
x_{1} a_{1}+x_{2} a_{2}=r+m\left(x_{1}, x_{2}\right) a_{3},
$$


или

$$
x_{1} a_{1}+x_{2} a_{2} \equiv r\left(\bmod a_{3}\right),
$$

где $0 \leqslant r<a_{3}$. Так как $a_{1}, a_{3}$ взаимно просты, существует $a_{1}^{-1}, 1 \leqslant a_{1}^{-1}<a_{3}$, для которого $a_{1} a_{1}^{-1} \equiv 1\left(\bmod a_{3}\right)$. Из оценок сумм Клостермана легко следует, что для любых фиксированных $0<\alpha_{1}<\alpha_{2}<1$ и $N \alpha_{1} \leqslant a_{1} \leqslant N \alpha_{2}$ обратный элемент $a_{1}^{-1}$ асимптотически равномерно распределен на $\left[1, \ldots, a_{3}\right]$. По-видимому, это также верно в нашем ансамбле. Перепишем (3) в виде

$$
x_{1}+a_{12} x_{2} \equiv r_{1}\left(\bmod a_{3}\right),
$$

где $r_{1} \equiv r a_{1}^{-1}\left(\bmod a_{3}\right), a_{12} \equiv a_{1}^{-1} a_{2}\left(\bmod a_{3}\right)$ и

$$
a_{12} x_{2} \equiv\left(r_{1}-x_{1}\right)\left(\bmod a_{3}\right)
$$

Уравнение (5) имеет естественную геометрическую интерпретацию. Рассмотрим $S=\left[0,1, \ldots, a_{3}-1\right]$ как "дискретную окружность". Сдвиг $R$ на $a_{12}$ $\left(\bmod a_{3}\right)$ есть вращение окружности $S$, а $\left\{a_{12} x_{2}\right\}$ есть орбита точки нуль под действием $R$. Тогда (5) означает, что $r_{1}-x_{1}$ принадлежит этой орбите.

Из леммы 1 получаем, что

$$
\begin{aligned}
F_{1}(a) & =\max _{r} \min _{\substack{x_{1} a_{1}+x_{2} a_{2} \equiv r\left(\bmod a_{3}\right) \\
0 \leqslant x_{1}, x_{2}<a_{3}}}\left(x_{1} a_{1}+x_{2} a_{2}\right) \\
& =N^{3 / 2} \max _{r} \min _{x_{1} a_{1}+x_{2} a_{2} \equiv r}\left(\bmod a_{3}\right) \\
& =N^{3 / 2} \max _{r_{1}} \min _{x_{1}+x_{2} a_{12} \equiv r_{1}}\left(\frac{x_{1}}{\sqrt{N}} \frac{a_{1}}{N}+\frac{x_{2}}{\sqrt{N}} \frac{a_{2}}{N}\right) \\
& \left(\frac{x_{1}}{\sqrt{N}} \frac{a_{1}}{N}+\frac{x_{2}}{\sqrt{N}} \frac{a_{2}}{N}\right) .
\end{aligned}
$$

Сначала мы локализуем наш ансамбль. Выберем $\alpha=\left(\alpha_{1}, \alpha_{2}, \alpha_{3}\right), 0<$ $\alpha_{j}<1, j=1,2,3, \varepsilon>0$ и определим $\Omega_{N, \alpha, \varepsilon} \subset \Omega_{N}$ как множество таких $a=\left(a_{1}, a_{2}, a_{3}\right)$, для которых $\left|\frac{a_{j}}{N}-\alpha_{j}\right| \leqslant \varepsilon$. Обозначим $P_{N, \alpha, \varepsilon}$ равномерное распределение вероятностей на $\Omega_{N, \alpha, \varepsilon}$. Теорема 1 будет установлена, если мы докажем ее по отношению к распределению $P_{N, \alpha, \varepsilon}$ (см. также конец этого параграфа). В ансамбле $\Omega_{N, \alpha, \varepsilon}$ отношения $\frac{a_{1}}{N}, \frac{a_{2}}{N} \varepsilon$-близки к $\alpha_{1}, \alpha_{2}$.

Мы используем некоторые факты из теории цепных дробей и теории вращения окружности (см. [6]). Возьмем число $\rho=\frac{a_{12}}{a_{3}}$ и разложим его в цепную дробь:

$$
\rho=\frac{1}{h_{1}+\frac{1}{h_{2}+\frac{1}{h_{3}+\ddots}+\frac{1}{h_{s_{0}}}}},
$$


где $h_{j} \geqslant 1$ - целые числа. Пусть

$$
\rho_{s}=\frac{1}{h_{1}+\frac{1}{h_{2}+\ddots \cdot+\frac{1}{h_{s}}}}=\frac{p_{s}}{q_{s}}
$$

- $s$-е приближение числа $\rho$. Можно найти "нечетные" интервалы $\Delta_{1}^{(2 p-1)}=$ $\left\{0,1, \ldots, m_{2 p-1}\right\}$ и "четные" интервалы $\Delta_{1}^{(2 p)}=\left\{a_{3}-m_{2 p}, \ldots, a_{3}-1\right\}, p \geqslant 1$, такие, что если $\Delta_{j}^{(2 p-1)}=R^{j} \Delta_{1}^{(2 p-1)}, \Delta_{j_{1}}^{(2 p)}=R^{j_{1}} \Delta_{1}^{(2 p)}$, то интервалы $\Delta_{j}^{(2 p-1)}$, $0 \leqslant j<q_{2 p}$, и $\Delta_{j_{1}}^{(2 p)}, 0 \leqslant j_{1}<q_{2 p-1}$, попарно не пересекаются и их объединение есть вся окружность $S$. Это значит, что $\Delta_{j}^{(2 p-1)}, \Delta_{j}^{(2 p)}$ образуют некоторое разбиение окружности $S$, которое мы обозначим $\eta^{(p)}$. Разбиения $\eta^{(p)}$ возрастают: $\eta^{(p+1)} \geqslant \eta^{(p)}$. Их точная структура зависит от структуры цепной дроби (7).

Мы покажем (см. ниже), что в (6) достаточно рассмотреть $x_{1} \leqslant \mathcal{D}_{1} \sqrt{N}$, $x_{2} \leqslant \mathcal{D}_{1} \sqrt{N}$, где $\mathcal{D}_{1}$ достаточно велико и зависит от $\rho$. Возьмем такое $s_{1}$, что $q_{s_{1}-1} \leqslant \sqrt{N}<q_{s_{1}}$. Если $x_{2}>\mathcal{D}_{1} \sqrt{N}$, то выберем $k_{1}$ так, что $q_{s_{1}+k_{1}} \leqslant x_{2}<$ $q_{s_{1}+k_{1}+1}$. Ясно, что $k_{1}$ растет, если $\mathcal{D}_{1}$ растет. Положим

$$
\begin{aligned}
& x_{1}^{\prime}=x_{1}+\left(a_{12} q_{s_{1}+k_{1}}-p_{s_{1}+k_{1}} a_{3}\right)=x_{1}+a_{3}\left(\rho q_{s_{1}+k_{1}}-p_{s_{1}+k_{1}}\right), \\
& x_{2}^{\prime}=x_{2}-q_{s_{1}+k_{1}} .
\end{aligned}
$$

Легко видеть, что

$$
x_{1}^{\prime}+a_{12} x_{2}^{\prime} \equiv x_{1}+a_{12} x_{2}\left(\bmod a_{3}\right)
$$

и

$$
\begin{aligned}
\frac{x_{1}^{\prime}}{\sqrt{N}} \frac{a_{1}}{N}+\frac{x_{2}^{\prime}}{\sqrt{N}} \frac{a_{2}}{N}= & \frac{x_{1}}{\sqrt{N}} \frac{a_{1}}{N}+\frac{x_{2}}{\sqrt{N}} \frac{a_{2}}{N} \\
& +\frac{a_{3}\left(\rho q_{s_{1}+k_{1}}-p_{s_{1}+k_{1}}\right)}{\sqrt{N}} \frac{a_{1}}{N}-\frac{q_{s_{1}+k_{1}}}{\sqrt{N}} \frac{a_{2}}{N} .
\end{aligned}
$$

Выражение

$$
\frac{a_{3}\left(\rho q_{s_{1}+k_{1}}-p_{s_{1}+k_{1}}\right)}{\sqrt{N}} \frac{a_{1}}{N}=\frac{a_{3}}{N} \sqrt{N}\left(\rho q_{s_{1}+k_{1}}-p_{s_{1}+k_{1}}\right) \frac{a_{1}}{N}
$$

убывает при возрастании $k_{1}$, поскольку $\left(\rho q_{s_{1}+k_{1}}-p_{s_{1}+k_{1}}\right)$ ведет себя как $\frac{1}{q_{s_{1}+k_{1}}}$. $\mathrm{C}$ другой стороны, $\frac{q_{s_{1}+k_{1}}}{\sqrt{N}}$ принимает значения порядка $O(1)$ и возрастает с ростом $k_{1}$. Следовательно, сумма двух последних членов в (8) становится отрицательной при достаточно большом $k_{1}$. Так как в (6) нас интересуют минимальные значения по $x_{2}^{\prime}$, выражение (6) принимает меньшее значение при $x_{2}^{\prime}=x_{2}-q_{s_{1}+k_{1}}$. Таким образом, достаточно рассмотреть $x_{2} \leqslant \mathcal{D}_{1} \sqrt{N}$ при достаточно большой постоянной $\mathcal{D}_{1}$, зависящей от $\rho$.

Покажем, что $x_{2}>\mathcal{D}_{2}^{-1} \sqrt{N}$ для некоторой достаточно большом $\mathcal{D}_{2}$. В самом деле, выберем $k_{2}$ так, чтобы $q_{s_{1}-k_{2}}<\mathcal{D}_{2}^{-1} \sqrt{N} \leqslant q_{s_{1}-k_{2}+1}$, и рассмотрим 
разбиение $\eta^{\left(s_{1}-k_{2}\right)}$. Возьмем любой элемент $\Delta=\left[y_{1}, y_{2}\right]$ этого разбиения. Тогда для $r_{1}=y_{2}-1$ значения $x_{1}$ равны

$$
\left(y_{2}-y_{1}-1\right), \quad\left(y_{2}-y_{1}-1\right)+\ell_{1}, \quad\left(y_{2}-y_{1}-1\right)+\ell_{1}+\ell_{2}, \ldots,
$$

где $\ell_{1}, \ell_{2}, \ldots$ - длины элементов разбиения $\eta^{\left(s_{1}-k_{2}\right)}$, следующих за $\Delta$. Если $x_{2} \leqslant \mathcal{D}_{2}^{-1} \sqrt{N} \leqslant q_{s_{1}-k_{2}+1}$, то ясно, что $\min \left(\frac{x_{1}}{\sqrt{N}} \frac{a_{1}}{N}+\frac{x_{2}}{\sqrt{N}} \frac{a_{2}}{N}\right)$ достигается в точке $x_{1}=y_{2}-y_{1}-1$. С другой стороны, рассмотрим для $r_{1}$ элемент $\Delta^{\prime}$ разбиения $\eta^{\left(s_{1}\right)}$, содержащий $r_{1}$. Возьмем $x_{1}^{\prime}=\left|\Delta^{\prime}\right|-1$. Ясно, что $x_{2}^{\prime} \leqslant q_{s_{1}+1}$ и $\frac{x_{1}^{\prime}}{\sqrt{N}} \frac{a_{1}}{N}+\frac{x_{2}^{\prime}}{\sqrt{N}} \frac{a_{2}}{N}$ много меньше, чем в предыдущем случае. Следовательно, $\mathcal{D}_{2}^{-1} \sqrt{N} \leqslant x_{2} \leqslant \mathcal{D}_{1} \sqrt{N}$.

В упомянутой выше статье К. Ульчиграй и второго автора [5] рассмотрена следующая задача. Возьмем большое $R$ и некоторое фиксированное число $k$. Для любого иррационального числа $\rho$ рассмотрим $q_{s}$ такие, что $q_{s-1} \leqslant R<q_{s}$, и $h_{s-k}, \ldots, h_{s}, \ldots, h_{s+k}$. В [5] доказано, что по отношению к гауссовской плотности $\frac{1}{(\ln 2)(1+\rho)}$ существует совместное предельное распределение величин $\frac{q_{s-1}}{R}$, $\frac{q_{s}}{R}, h_{s-k}, \ldots, h_{s}, \ldots, h_{s+k}$. По-видимому, то же самое предельное распределение возникает для любого вероятностного распределения $P_{N, \alpha, \varepsilon}$, но мы не рассматриваем более детально этот вопрос.

Для любых $s$ и $k$ рассмотрим элементы $\Delta^{\prime}, \Delta^{\prime \prime}$ разбиения $\eta^{(s-k)}$, содержащие 0 (для $\Delta^{\prime}$ точка 0 является правым концом, а для $\Delta^{\prime \prime}$ она является левым концом). Разбиения $\eta^{(s-k+1)}, \eta^{(s-k+2)}, \ldots, \eta^{(s+k)}$ порождают конечное разбиение объединения $\Delta^{\prime} \cup \Delta^{\prime \prime}$, которое мы обозначим $\nu$. Структура этого разбиения определяется значениями $h_{s-k}, h_{s-k+1}, \ldots, h_{s+k}$. Обозначим через $N$ конечное множество концевых точек элементов разбиения $\nu$.

Возьмем $b=a_{3}-a_{12}$ и $N^{\prime} \equiv N-a_{12}\left(\bmod a_{3}\right)$. Обозначим

$$
f_{k}=\max _{y \in N^{\prime}} \min _{\substack{x_{1}+a_{12} x_{2} \equiv y\left(\bmod a_{3}\right) \\ a_{12} x_{2} \in N^{\prime}}}\left(\frac{x_{1}}{\sqrt{N}} \frac{a_{1}}{N}+\frac{x_{2}}{\sqrt{N}} \frac{a_{2}}{N}\right) .
$$

Те же самые аргументы, что и прежде, показывают, что с $P_{N, \alpha, \varepsilon}$-вероятностью, стремящейся к 1 при $k \rightarrow \infty$, решение основной "max-min"-задачи для $F_{1}$ дается с помощью $f_{k}$. В этом смысле оно является функцией от $\frac{q_{s-1}}{R}, \frac{q_{s}}{R}$ и $h_{s-k}, \ldots, h_{s+k}$ и имеет предельное распределение при $N \rightarrow \infty$.

\section{§ 3. Случай $n=3$ и произвольных $b_{i j}=$ нод $\left(a_{i}, a_{j}\right)$}

Так как все $a_{j}$ не имеют общих делителей, то $b_{13}$ и $b_{23}$ взаимно просты. Снова для заданного $r, 0 \leqslant r<a_{3}$, мы рассмотрим уравнение

$$
x_{1} a_{1}+x_{2} a_{2}=r+m\left(x_{1}, x_{2}\right) a_{3} .
$$


Напишем $a_{1}=b_{13} a_{1}^{\prime}, a_{2}=b_{23} a_{2}^{\prime}, a_{3}=b_{13} b_{23} a_{3}^{\prime}$. Ясно, что пары $\left(a_{1}^{\prime}, a_{3}^{\prime}\right)$, и $\left(a_{2}^{\prime}, a_{3}^{\prime}\right)$ взаимно простые. Пусть

$$
\begin{aligned}
r & =r^{\prime} b_{13} b_{23}+r^{\prime \prime}, & & 0 \leqslant r^{\prime \prime}<b_{13} b_{23}, \\
x_{1} & =b_{23} x_{1}^{\prime}+x_{1}^{\prime \prime}, & & 0 \leqslant x_{1}^{\prime \prime}<b_{23}, \\
x_{2} & =b_{13} x_{2}^{\prime}+x_{2}^{\prime \prime}, & & 0 \leqslant x_{2}^{\prime \prime}<b_{13}, \\
a_{1}^{\prime} & =b_{23} a_{1}^{\prime \prime}+a_{1}^{\prime \prime \prime}, & & 0 \leqslant a_{1}^{\prime \prime \prime}<b_{23}, \\
a_{2}^{\prime} & =b_{13} a_{2}^{\prime \prime}+a_{2}^{\prime \prime \prime}, & & 0 \leqslant a_{2}^{\prime \prime \prime}<b_{13} .
\end{aligned}
$$

Сначала рассмотрим уравнение

$$
x_{1}^{\prime \prime} b_{13} a_{1}^{\prime \prime \prime}+x_{2}^{\prime \prime} b_{23} a_{2}^{\prime \prime \prime} \equiv r^{\prime \prime}\left(\bmod b_{13} b_{23}\right) .
$$

Мы можем найти единственное решение, которое мы обозначим $\bar{x}_{1}^{\prime \prime}, \bar{x}_{2}^{\prime \prime}$, такое, что

$$
x_{1}^{\prime \prime} a_{1}^{\prime \prime \prime}+x_{2}^{\prime \prime} a_{2}^{\prime \prime \prime}=r^{\prime \prime}+t b_{13} b_{23},
$$

где $t$ может принимать значения 0 или 1. Затем мы рассмотрим уравнение, которое получается после деления обеих частей уравнения (3) на $b_{13} b_{23}$ :

$$
x_{1}^{\prime} a_{1}^{\prime}+a_{2}^{\prime} a_{2}^{\prime}=r^{\prime}-x_{1}^{\prime \prime} a_{1}^{\prime \prime}-x_{2}^{\prime \prime} a_{2}^{\prime \prime}-t+m a_{3}^{\prime} .
$$

Положим $r_{1}^{\prime}=r^{\prime}-x_{1}^{\prime \prime} a_{1}^{\prime \prime}-x_{2}^{\prime \prime} a_{2}^{\prime \prime}+t$. Ясно, что $x_{1}^{\prime \prime} a_{1}^{\prime \prime}-x_{2}^{\prime \prime} a_{2}^{\prime \prime}+t$ может принимать конечное число значений, зависящее только от $\left\{b_{i j}\right\}$. Легко видеть, что пределы вероятностей этих значений существуют при $N \rightarrow \infty$.

Уравнение (9) подобно (6), так как $a_{1}^{\prime}$ и $a_{3}^{\prime}$ взаимно просты. Мы можем написать

$$
x_{1}^{\prime}+x_{2}^{\prime} a_{2}^{\prime}\left(a_{1}^{\prime}\right)^{-1}=r_{1}^{\prime}\left(a_{1}^{\prime}\right)^{-1}+m_{1} a_{3}^{\prime}
$$

и использовать те же самые аргументы, что в $\S 2$. В частности, мы рассмотрим разложение $a_{2}^{\prime}\left(a_{1}^{\prime}\right)^{-1}$ в цепную дробь, возьмем $s_{1}$, для которого $q_{s_{1}} \geqslant \sqrt{N}$, $q_{s_{1}}-1<\sqrt{N}$, и найдем значение $s$, при котором $\frac{\left|\Delta_{1}^{(s)}\right|}{\sqrt{N}}+\frac{q_{s}}{\sqrt{N}}$ достигает своего минимума. Предельное распределение последнего выражения дает предельное распределение величины $\frac{1}{N^{3 / 2}} F_{1}(a)$.

\section{§4. Случай $n>3$}

Для $n>3$ наш результат более слабый. Снова рассмотрим уравнение

$$
x_{1} a_{1}+x_{2} a_{2}+\cdots+x_{n-1} a_{n-1}=r+m a_{n},
$$

или

$$
x_{1} a_{1}+x_{2} a_{2}+\cdots+x_{n-1} a_{n-1} \equiv r\left(\bmod a_{n}\right) .
$$

Левая часть уравнения - это орбита абелевой группы, порожденной $(n-1)$ коммутирующими вращениями $R_{j}$, где $R_{j}-$ сдвиг $\bmod a_{n}$ окружности $S=$ $\left\{0,1, \ldots, a_{n}-1\right\}$ на $a_{j}, 1 \leqslant j \leqslant n-1$. Мы докажем следующую теорему. 
Теорема 2. Рассмотрим ансамбль $\Omega_{N, \alpha} \subset \Omega_{N}$ такой, что $\alpha N<a_{j}, 1 \leqslant$ $j \leqslant n$, где $0<\alpha<1-\oint$ - ксированное число. Пусть $\Sigma_{\mathcal{D}} \subset \Omega_{N, \alpha}-$ множество таких наборов $\left(a_{1}, a_{2}, \ldots, a_{n}\right) \in \Omega_{N, \alpha}$, что для любого $r \in S$ уравнение $(12)$ имеет решение с $0 \leqslant x_{j} \leqslant \mathcal{D} N^{\frac{1}{n-1}}$. Тогда $P_{N, \alpha}\left(\Sigma_{\mathcal{D}}\right) \geqslant 1-\varepsilon(\mathcal{D})$, где $\varepsilon(\mathcal{D}) \rightarrow 0$ при $\mathcal{D} \rightarrow \infty$. Здесъ $P_{N, \alpha}-$ равномерное распределение на $\Omega_{N, \alpha}$.

ДокАзАТЕЛьство. Легко видеть, что

$$
\frac{1}{a_{n}} \sum_{m=0}^{a_{n}-1} \exp \left\{-\frac{2 \pi i m r}{a_{n}}\right\} \exp \left\{-2 \pi i \sum_{j=1}^{n-1} \frac{m a_{j}}{a_{n}} x_{j}\right\}= \begin{cases}1, & \text { если (12) выполнено, } \\ 0 & \text { в противном случае. }\end{cases}
$$

Возьмем $M>0$ и рассмотрим вес на $\mathbb{Z}$

$$
c(x)= \begin{cases}1-\frac{|x-M|}{M}, & 0 \leqslant x \leqslant 2 M \\ 0 & \text { в противном случае. }\end{cases}
$$

Чтобы показать, что (12) имеет решение для любого $r \in S$, достаточно показать, что для любого $r$

$$
\begin{aligned}
Z_{a}(r)= & \sum_{x_{1}, x_{2}, \ldots, x_{n} \in \mathbb{Z}} c\left(x_{1}\right) c\left(x_{2}\right) \cdots c\left(x_{n-1}\right) \frac{1}{a_{n}} \sum_{m=0}^{a_{n}-1} \exp \left\{-\frac{2 \pi i m r}{a_{n}}\right\} \\
& \times \exp \left\{2 \pi i m \sum_{j=1}^{n-1} \frac{a_{j}}{a_{n}} x_{j}\right\} \neq 0 .
\end{aligned}
$$

Перепишем предыдущее выражение в виде

$$
Z_{a}(r)=\frac{1}{a_{n}} \sum_{m=0}^{a_{n}-1} \exp \left\{-2 \pi i \frac{m r}{a_{n}}\right\} \prod_{j=1}^{n-1} \sum_{x \in \mathbb{Z}} c(x) \exp \left\{2 \pi i \frac{m a_{j}}{a_{n}} x\right\} .
$$

Легко проверить, что

$$
\sum_{x \in \mathbb{Z}} c(x) e^{-2 \pi i \theta x}=e^{2 \pi i M \theta} \frac{1}{2 M+1}\left\{\frac{\sin \pi(2 M+1) \theta}{2 \sin \pi \theta}\right\}^{2}
$$

для любых $\theta$. Разделяя в (13) вклад от $m=0$ и от $m \neq 0$, мы можем написать

$$
Z_{a}(r)=\frac{M^{n-1}}{a_{n}}+Z_{a}^{(1)}(r)=\frac{M^{n-1}}{a_{n}}+\frac{1}{a_{n}} \sum_{m=1}^{a_{n}-1} e^{-2 \pi i \frac{m r}{a_{n}}} \prod_{j=1}^{n-1}\left(\sum_{x \in \mathbb{Z}} c(x) e^{2 \pi i \frac{m a_{j}}{a_{n}} x}\right) .
$$

Рассмотрим $M=A N^{\frac{1}{n-1}}$. Тогда $\frac{M^{n-1}}{a_{n}}=\frac{A^{n-1} N}{a_{n}}$. В нашем случае $\alpha \leqslant \frac{a_{n}}{N} \leqslant 1$. Ввиду (14) для $Z_{a}^{(1)}(r)$ мы имеем оценку

$$
\left|Z_{a}^{(1)}(r)\right| \leqslant \frac{1}{a_{n}} \sum_{m=1}^{a_{n}-1} \prod_{j=1}^{n-1}\left(\frac{\sin \pi(2 M+1) \theta_{j}}{2 \sin \pi \theta_{j}}\right)^{2} \frac{1}{2 M+1},
$$

где $\theta_{j}=\frac{m a_{j}}{a_{n}}$. Эта оценка не зависит от $r$. Поэтому, если мы покажем, что математическое ожидание правой части в (15) ограничено некоторой постоянной, то утверждение теоремы будет легко вытекать из неравенства Чебышёва. 
Легко проверить, что

$$
\left(\frac{\sin \pi(2 M+1) \theta_{j}}{2 \sin \pi \theta_{j}}\right)^{2} \leqslant \frac{C_{1}}{\left(\sin \left(\pi m a_{j} / a_{n}\right)\right)^{2}+M^{-2}}
$$

для некоторой абсолютной постоянной $C_{1}$. Следовательно,

$$
\begin{aligned}
\left|Z_{a}^{(1)}(r)\right| & \leqslant \frac{C_{2}}{a_{n}} \sum_{m=1}^{a_{n}-1} \prod_{j=1}^{n-1}\left\{\frac{1}{M} \frac{1}{\left(\sin \left(\pi m a_{j} / a_{n}\right)\right)^{2}+M^{-2}}\right\} \\
& \leqslant \frac{C_{2}}{a_{n}} \sum_{m=1}^{a_{n}-1} \prod_{j=1}^{n-1} \frac{1}{M\left\|m a_{j} / a_{n}\right\|^{2}+M^{-1}}
\end{aligned}
$$

для другой абсолютной постоянной $C_{2}$. В последней формуле $\|\cdot\|$ - расстояние до ближайшего целого числа. Усредним правую часть (16) по мере $P_{N, \alpha}$, т. е. рассмотрим

$$
Z_{a}^{(2)}(r)=\frac{1}{N^{n+1}} \sum_{\alpha N \leqslant a_{n} \leqslant N} \sum_{m=1}^{a_{n}-1} \sum_{\substack{\alpha N \leqslant a_{1}, \ldots, a_{n-1} \leqslant N \\ \text { нод }\left(a_{1}, \ldots, a_{n}\right)=1}} \prod_{j=1}^{n-1} \frac{1}{M\left\|m a_{j} / a_{n}\right\|^{2}+M^{-1}} .
$$

Предположим, что $\frac{m}{a_{n}}=\frac{b}{q}$ для некоторого $1 \leqslant b \leqslant q$ и нод $(b, q)=1$. Тогда правая часть (17) может быть записана в виде

$$
N^{-n-1} \sum_{q=1}^{N} \frac{N}{q} \sum_{\substack{1 \leqslant b \leqslant q \\ \text { нод }(b, q)=1}} \sum_{\substack{\alpha \leqslant a_{1}, \ldots, a_{n} \\ \text { нод }\left(a_{1}, \ldots, a_{n-1}, q\right)=1}} \prod_{j=1}^{n-1} \frac{1}{M\left\|\frac{b}{q} a_{j}\right\|^{2}+M^{-1}} .
$$

Если нод $\left(a_{1}, \ldots, a_{n-1}, q\right)$ равен единице, то $a_{k}$ не делится на $q$ для некоторых $k=1, \ldots, n-1$. Поэтому

$$
\begin{aligned}
\sum_{\alpha N \leqslant a_{1}, \ldots, a_{n} \leqslant N} & \prod_{j=1}^{n-1} \frac{1}{M\left\|\frac{b}{q} a_{j}\right\|^{2}+M^{-1}} \\
& \leqslant \sum_{k=1}^{n-1} \sum_{\substack{\alpha \leqslant a_{1}, \ldots, a_{n-1} \leqslant N \\
a_{k} \notin q \mathbb{Z}}} \prod_{j=1}^{n-1} \frac{1}{M\left\|\frac{b}{q} a_{j}\right\|^{2}+M^{-1}} \\
& =(n-1) \sum_{\substack{\alpha N \leqslant a \leqslant N \\
a \neq q \mathbb{Z}}} \frac{1}{M\left\|\frac{b}{q} a\right\|^{2}+M^{-1}} \cdot\left(\sum_{\alpha N \leqslant a \leqslant N} \frac{1}{M\left\|\frac{b}{q} a\right\|^{2}+M^{-1}}\right)^{n-2} .
\end{aligned}
$$

Теперь мы оценим обе суммы в (19).

Лемма 2. Имеют место неравенства:

(i) если $q>M$, mo

$$
\sum_{\alpha N \leqslant a \leqslant N} \frac{1}{M\left\|\frac{b}{q} a\right\|^{2}+M^{-1}} \leqslant N
$$


(ii) если $q \leqslant M$, mo

$$
\sum_{\alpha N \leqslant a \leqslant N} \frac{1}{M\left\|\frac{b}{q} a\right\|^{2}+M^{-1}} \leqslant \frac{M}{q} N
$$

(iii) если $q \leqslant M$, mo

$$
\sum_{\substack{\alpha \leqslant a \leqslant N \\ a \notin q \mathbb{Z}}} \frac{1}{M\left\|\frac{b}{q} a\right\|^{2}+M^{-1}} \leqslant \frac{q}{M} N
$$

ДокАЗАтельство. Разобьем $[\alpha N, N]$ на приблизительно $\frac{(1-\alpha) N}{q}$ интервалов $I_{\gamma}$ длины $q$. Обозначим через $\pi_{q}$ факторотображение $\mathbb{Z} \rightarrow \mathbb{Z} / q \mathbb{Z}$. Тогда для каждого $I_{\gamma}$ мы имеем $\left\{\pi_{q}(b a) \mid a \in I_{\gamma}\right\}=\mathbb{Z}_{q}$ и

$$
\left\{\pi_{q}(b a) \mid a \in I_{\gamma}, \pi_{q}(a) \neq 0\right\}=\mathbb{Z}_{q} \backslash\{0\} .
$$

Следовательно, сумма

$$
\sum_{\alpha N \leqslant a \leqslant N} \frac{1}{M\left\|\frac{b}{q} a\right\|^{2}+M^{-1}}
$$

ведет себя как

$$
\frac{N}{q} \sum_{z=0}^{q-1}(q-1) \frac{1}{M\left\|\frac{z}{q}\right\|^{2}+M^{-1}}
$$

и для абсолютной постоянной $C_{3}$

$$
\sum_{z=0}^{q-1} \frac{1}{M\left\|\frac{z}{q}\right\|^{2}+M^{-1}} \leqslant C_{3} q, \quad \text { если } q \geqslant M,
$$

и

$$
\sum_{z=0}^{q-1} \frac{1}{M\left\|\frac{z}{q}\right\|^{2}+M^{-1}} \leqslant C_{3} M, \quad \text { если } \quad q<M .
$$

Из этого получаем утверждения (i) и (ii) леммы. Для $q<M$ сумма

$$
\sum_{z=1}^{q-1} \frac{1}{M\left\|\frac{z}{q}\right\|^{2}+M^{-1}} \leqslant \frac{q^{2}}{M} .
$$

Лемма 2 доказана.

Вернемся к (19). Для $q \geqslant M$ лемма 2 показывает, что правая часть не превосходит $N^{n-1}$, а для $q<M-$ не превосходит $\frac{q}{M} N\left(\frac{M}{q} N\right)^{n-2}=\left(\frac{M}{q}\right)^{n-3} N^{n-1}$. Подставляя эти оценки в (17), мы получаем в предположении $n>3$, что

$$
\begin{aligned}
Z_{a}^{(2)}(r) & \leqslant \frac{1}{N^{n+1}} \sum_{M \leqslant q \leqslant N} \frac{N}{q} \sum_{\substack{1 \leqslant b \leqslant q \\
(b, q)=1}} N^{n-1}+\frac{1}{N^{n+1}} \sum_{1 \leqslant q \leqslant M} \frac{N}{q} \sum_{\substack{1 \leqslant b \leqslant q \\
(b, q)=1}}\left(\frac{M}{q}\right)^{n-3} \cdot N^{n-1} \\
& <C_{4}+C_{4} \frac{M^{n-3}}{N} \sum_{1 \leqslant q \leqslant M} \frac{1}{q^{n-3}}<C_{4}\left(1+\frac{M^{n-2}}{N}\right)
\end{aligned}
$$


для другой константы $C_{4}$. Следовательно, $Z_{a}^{(2)}(r)$ ограничены. Из этого вытекает утверждение теоремы 2.

Теорема 2 показывает, что для любого ансамбля $\Omega_{N, \alpha}$ семейство вероятностных распределений величины $F_{1}(a) / N^{1+\frac{1}{n-1}}$ слабо компактно. Однако из этого не следует существование предельного распределения для $F_{1}(a) / N^{1+\frac{1}{n-1}}$, а следует только существование предельных точек.

\section{Приложение}

Ниже мы доказываем оценку, которая не используется в предыдущих доказательствах, но представляет некоторый независимый интерес. Подобное утверждение было доказано А. Кочергиным (устное сообщение).

Лемма. Для $0<\alpha<1$ положим

$$
S_{T}(\alpha)=\frac{1}{T} \sum_{t=1}^{T} \frac{1}{|\exp \{2 \pi i t \alpha\}-1|}
$$

u

$$
A_{T}(\mathcal{D})=\left\{\alpha:\left|S_{T}(\alpha)\right| \geqslant \mathcal{D} \ln T\right\} .
$$

Тогда лебегова мера $\ell\left(A_{T}(\mathcal{D})\right) \leqslant \varepsilon_{1}(\mathcal{D})$, где $\varepsilon_{1}(\mathcal{D}) \rightarrow 0$ при $\mathcal{D} \rightarrow \infty$.

ДокАЗАТЕЛьство. Возьмем два положительных числа $C_{1}, C_{2}, 1<C_{1}<$ $2 C_{1}<C_{2}$, и определим интервалы

$$
\Delta_{T}(k)=\left\{\alpha: \frac{C_{1}^{k}}{T} \leqslant \alpha \leqslant \frac{C_{1}^{k+1}}{T}\right\}, \quad k=0,1, \ldots, K .
$$

Без потери общности мы можем предположить, что $C_{1}^{K+1}=T$. Ясно, что $K \sim \frac{\ln T}{\ln C_{1}}$. Рассмотрим

$$
B_{T, k}\left(C_{1}, C_{2}\right)=\left\{\alpha: \nu_{T, k}(\alpha) \leqslant C_{2} C_{1}^{k}\right\}
$$

где $\nu_{M, k}(\alpha)$ - число тех $m, 1 \leqslant m \leqslant M$, для которых $\{m \alpha\} \in \Delta_{M}(k)$, и

$$
B_{T}\left(C_{1}, C_{2}\right)=\bigcap_{k=0}^{K} B_{T, k}\left(C_{1}, C_{2}\right)
$$

Для $\alpha \in B_{T}\left(C_{1}, C_{2}\right)$ мы имеем

$$
\begin{aligned}
\left|S_{T}(\alpha)\right| & =\frac{1}{T} \sum_{t=1}^{T} \frac{1}{|\exp \{2 \pi i t \alpha\}-1|} \leqslant \frac{1}{T} \sum_{k=0}^{K} \frac{T}{2 \pi C_{1}^{k}} \nu_{T, k}(\alpha) \\
& \leqslant \frac{C_{2}}{2 \pi}(K+1) \leqslant \frac{C_{2} \ln T}{2 \pi \ln C_{1}} .
\end{aligned}
$$


Это есть требуемое неравенство с $\mathcal{D}=\frac{C_{2}}{2 \pi \ln C_{1}}$. Следовательно, мы должны оценить меру дополнения к $B_{T}\left(C_{1}, C_{2}\right)$. Ясно, что

$$
\ell\left(\bar{B}_{T}\left(C_{1}, C_{2}\right)\right) \leqslant \sum_{k=0}^{K} \ell\left(\bar{B}_{T, k}\left(C_{1}, C_{2}\right)\right)
$$

где $\bar{B}$ - дополнение к $B$. Пусть $\chi_{k}(\alpha)$ - индикатор множества $B_{T, k}\left(C_{1}, C_{2}\right)$. Тогда

$$
\nu_{T, k}\left(C_{1}, C_{2}\right)=\sum_{t=1}^{T} \chi_{k}(t \alpha)
$$

и по неравенству Чебышёва

$$
\begin{aligned}
\ell\left\{\alpha: \nu_{T, k}(\alpha) \geqslant C_{2} C_{1}^{k}\right\} & =\ell\left\{\alpha: \sum_{t=1}^{T} \chi_{k}(t \alpha) \geqslant C_{2} C_{1}^{k}\right\} \\
& =\ell\left\{\alpha: \sum_{t=1}^{T}\left(\chi_{k}(t \alpha)-\frac{C_{1}^{k}\left(C_{1}-1\right)}{T}\right) \geqslant\left(C_{2}-C_{1}+1\right) C_{1}^{k}\right\} \\
& \leqslant \frac{\mathrm{E}\left[\sum_{t=1}^{T}\left(\chi_{k}(t \alpha)-\frac{C_{1}^{k}\left(C_{1}-1\right)}{T}\right)^{2}\right]}{\left(C_{2}-C_{1}+1\right)^{2} C_{1}^{2 k}} \\
& =\frac{\sum_{j=1}^{T}(T-j)\left[\mathrm{E} \chi_{k}(\alpha) \chi_{k}(j \alpha)-\left(\frac{C_{1}^{k}\left(C_{1}-1\right)}{T}\right)^{2}\right]}{\left(C_{2}-C_{1}+1\right)^{2} C_{1}^{2 k}} .
\end{aligned}
$$

Здесь математическое ожидание берется относительно меры Лебега. Мы оценим последнюю сумму. Это будет сделано в четыре шага.

$$
\begin{aligned}
& \text { Шаг 1: } j<C_{1} \text {. Здесь } \mathrm{E} \chi_{k}(\alpha) \chi_{k}(j \alpha) \leqslant \mathrm{E} \chi_{k}(\alpha)=\frac{C_{1}^{k}\left(C_{1}-1\right)}{T} \text { и } \\
& \frac{\sum_{j<C_{1}}(T-j)\left[\mathrm{E} \chi_{k}(\alpha) \chi_{k}(j \alpha)-\left(\frac{C_{1}^{k}\left(C_{1}-1\right)}{T}\right)^{2}\right]}{\left(C_{2}-C_{1}+1\right)^{2} C_{1}^{2 k}} \leqslant \frac{C_{1}^{k}\left(C_{1}-1\right) C_{1}}{C_{1}^{2 k}\left(C_{2}-C_{1}+1\right)^{2}} \leqslant \frac{1}{C_{1}^{k+1}},
\end{aligned}
$$

так как $C_{2}>2 C_{1}$.

Шаг 2: $C_{1} \leqslant j<\frac{T}{C_{1}^{k+1}}$. В этом случае $\mathrm{E} \chi_{k}(\alpha) \chi_{k}(j \alpha)=0$ и не надо ничего оценивать. В самом деле, $\chi_{k}(\alpha)$ есть индикатор множества $\Delta_{T}(k)$. Функция $\chi_{k}(j \alpha)$ является индикатором интервала из арифметической прогрессии интервалов $\left[\frac{1}{j} \frac{C_{1}^{k}}{T}+\frac{s}{j}, \frac{1}{j} \frac{C_{1}^{k+1}}{T}+\frac{s}{j}\right], s \geqslant 0$. Из нашего условия на $j$ следует, что $\Delta_{T}(k)$ может пересекаться только с интервалом, для которого $t=0$; это пересечение пусто.

Шаг 3: $\frac{T}{C_{1}^{k+1}} \leqslant j<\frac{3 T}{C_{1}^{k}\left(C_{1}-1\right)}$. Число 3 не играет никакой существенной роли и может быть заменено любым большим числом. Здесь $\Delta_{T}(k)$ пересекается не более чем с тремя интервалами вышеупомянутой арифметической прогрессии. 
Следовательно, $\mathrm{E} \chi_{k}(\alpha) \chi_{k}(j \alpha) \leqslant \frac{3 C_{1}^{k}}{j T}$ и

$$
\begin{aligned}
& \frac{\sum_{j}(T-j)\left[\mathrm{E} \chi_{k}(\alpha) \chi_{k}(j \alpha)-\left(\frac{C_{1}^{k}\left(C_{1}-1\right)}{T}\right)^{2}\right]}{\left(C_{2}-C_{1}+1\right)^{2} C_{1}^{2 k}} \\
& \leqslant \frac{3 C_{1}^{k}}{\left(C_{2}-C_{1}+1\right)^{2} C_{1}^{2 k}} \sum_{\frac{T}{C_{1}^{k+1} \leqslant j \leqslant \frac{3 T}{C_{1}^{k}\left(C_{1}-1\right)}}} \frac{1}{j} \\
& \leqslant \frac{3}{C_{1}^{k}\left(C_{2}-C_{1}+1\right)^{2}} \frac{C_{1}^{k+1} \cdot 2 T}{T C_{1}^{k}\left(C_{1}-1\right)^{2}}=\frac{24}{C_{1}^{k} C_{2}} .
\end{aligned}
$$

Это и есть нужная оценка.

Шаг 4: $j \geqslant \frac{3 T}{C_{1}^{k}\left(C_{1}-1\right)}$. В этом случае $\mathrm{E} \chi_{k}(\alpha) \chi_{k}(j \alpha)$ близко к $\left(\mathrm{E} \chi_{k}(\alpha)\right)^{2}=$ $\left(\frac{C_{1}^{k}\left(C_{1}-1\right)}{T}\right)^{2}$. Действительно, мы можем увеличить $\Delta_{T}(k)$, добавив интервалы вблизи каждого конца так, чтобы новое множество $\Delta_{T}^{\prime}(k)$ состояло из целого числа интервалов $\left[\frac{s}{j}, \frac{s+1}{j}\right]$. Тогда

$$
\begin{aligned}
\mathrm{E} \chi_{k}(\alpha) \chi_{k}(j \alpha) & \leqslant \ell\left(\Delta_{T}^{\prime}(k)\right) \frac{C_{1}^{k}\left(C_{1}-1\right)}{T} \leqslant\left(\ell\left(\Delta_{T}(k)\right)+\frac{2}{j}\right) \frac{C_{1}^{k}\left(C_{1}-1\right)}{T} \\
& =\ell^{2}\left(\Delta_{T}(k)\right)+\frac{2 C_{1}^{k}\left(C_{1}-1\right)}{T j}
\end{aligned}
$$

и

$$
\begin{gathered}
\sum_{\frac{3 T}{C_{1}^{k}(C-1)} \leqslant j \leqslant T} \frac{(T-j)\left[\mathrm{E} \chi_{k}(\alpha) \chi_{k}(j \alpha)-\left(\frac{C_{1}^{k}\left(C_{1}-1\right)}{T}\right)^{2}\right]}{\left(C_{2}-C_{1}+1\right)^{2} C_{1}^{2 k}} \\
\leqslant \sum_{\frac{3 T}{C^{k}(C-1)} \leqslant j \leqslant T} \frac{(T-j) \cdot 2 C_{1}^{k}\left(C_{1}-1\right)}{T j\left(C_{2}-C_{1}+1\right)^{2} C_{1}^{2 k}} \\
\leqslant \frac{2 C_{1}^{k}\left(C_{1}-1\right)}{\left(C_{2}-C_{1}+1\right)^{2} C_{1}^{k}} \ln \frac{T C_{1}^{k}(C-1)}{3 T}=\frac{4\left(C_{1}-1\right) k \ln C_{1}}{\left(C_{2}-C_{2}+1\right)^{2} C_{1}^{k}} .
\end{gathered}
$$

Теперь мы можем закончить доказательство леммы. Из всех наших оценок следует, что

$$
\ell\left\{\alpha: \nu_{T, k}(\alpha) \geqslant C_{2} C_{1}^{k}\right\} \leqslant \frac{4 \ln C_{1}}{C_{1}} \frac{k}{C_{1}^{k}}
$$

и, значит,

$$
\sum_{k} \ell\left\{\alpha: \nu_{T, k}(\alpha) \geqslant C_{2} C_{1}^{k}\right\} \leqslant \operatorname{const} \frac{\ln C_{1}}{C_{1}}
$$

Отсюда вытекает утверждение леммы. 


\section{Список литературы}

[1] В.И. Арнольд, "Слабые асимптотики чисел решений диофантовых проблем", Функи. анализ и его прилож., 33:4 (1999), 65-66; англ. пер.: V. I. Arnold, "Weak asymptotics for the numbers of solutions of Diophantine problems", Funct. Anal. Appl., 33:4 (1999), 292-293.

[2] V.I. Arnold, "Arithmetical turbulence of selfsimilar fluctuations statistics of large Frobenius numbers of additive semigroups of integers", Mosc. Math. J., 7:2 (2007), 173-193.

[3] L. B. Koralov, Ya. G. Sinai, Probability theory and random processes, Springer-Verlag, 2007.

[4] J. J. Sylvester, "Mathematical questions with their solutions", Educational Times, 41 (1884), 21.

[5] Ya. G. Sinai, C. Ulcigrai, "Renewal-type limit theorem for the Gauss map and continued fractions", Ergodic Theory Dynam. Systems (to appear).

[6] Я.Г. Синай, Современные проблемы эргодической теории, Физматлит, М., 1995; пер. с англ.: Ya. G. Sinai, Topics in ergodic theory, Princeton Math. Ser., 44, Princeton Univ. Press, Princeton, NJ, 1994.

\section{Ж. Бургейн (J. Bourgain)}

Institute for Advanced Study, Princeton, USA

E-mail: bourgain@math.ias.edu

Я. Г. Синай (Ya. G. Sinai)

Princeton University, USA

E-mail: sinai@math.princeton.edu
Поступила в редакцию

29.05.2007 